\title{
An Efficient Human Body Contour Extraction Method for Mobile Apps
}

\author{
Li Wang ${ }^{1}$, Tao Ruan Wan ${ }^{2}$, Wen Tang ${ }^{3}$, Yao lin $\mathrm{Zhu}^{1}$, and Tong $\mathrm{Wu}^{1}$ \\ ${ }^{1}$ College of Electronic and Information, Xian Polytechnic University, Xian, China \\ 2 Faculty of Informatics, University of Bradford, Bradford, UK \\ 3 Faculty of Science and Technology, Bournemouth University,Bournemouth, UK
}

\begin{abstract}
With the increasing prevalence of powerful mobile technology, many applications involve human body measurements, such as online cloth shopping. Aiming at the application of non-contact human body measurement and modeling system, this paper presents a new method for extracting human contours in complex background environment. Since the H component on the HSV color space is independent of the light changes, the hair and the lower garment can be divided. Therefore, a method of using the elliptical skin model on YCbCr color space is proposed to detect the skin color, then, automatically extract the skin samples to determine the threshold segmentation range. The combination of the two improves the skin color point recognition rate, gradually separating the body skin, clot and the hair by using these binary values of the linear fusion operation to get the final body contours. Our experiments demonstrates that this algorithm effectively reduces constraints on background environment when extracting contours.
\end{abstract}

Keywords: Contour Extraction, Mobile Apps, Online Clothing, Gaussian Mixture Model, Threshold Segmentation, Linear Fusion

\section{Introduction}

The extraction of human contours is an essential part of the development of vision-based non-contact human body measurements and modeling systems. Applications of such systems are online shopping, online games, virtual reality applications. It directly affects the accuracy of subsequent body size measurements. Therefore, how to accurately extract human contours in images is a top priority [7]. Background environment directly affects the accuracy of human identification segmentation techniques.

Some research work makes a strict background requirements in order to extract accurate human body contours. Lin et al. [6] [4] collected images that were pretreated by re-edge detection to extract more accurate body contours. The requirement was that the person must wear special clothing to reduce the computation workload. Deng et al [3] have used the background difference method to extract the body contours based on the condition that all the pixels were independent, thus, a fixed camera with a good location on the characters and scenes 
was needed. Camera parameters and environmental conditions error are not conducive to universal. Yang et al.[13] proposed a body contour extraction method based on the HSV color space [10], which reduced the requirements for background, clothing, and lighting. However, for backdrops and floors, the method required a solid color and the color of clothing that were distinctive from the surrounding environment to segment the skin, clothes, floor, background wall for the extraction of body contours. But the method did not establish a skin model, because the range of the skin colour difference amongst different people can be very large, so the algorithm is not highly adaptable in different situations.

The main contribution of this paper is to develop a method for body contour extraction without too many restrictions, so that it is widely applicable to various conditions for online applications such as mobile applications (mobile apps.).

\section{Background}

A large number of studies have shown that the main factor in the appearance of human skin color differences is due to the chromaticity. The distribution of skin colors of different people is similar to an elliptical shape in a smaller area [9]. Currently, skin detection models can be roughly categorized into threshold based method [12] and elliptical models [9]. The threshold method determines the range of the color space component in order to distinguish between the skin color and non-skin color points, albeit it is fast and efficient, it is difficult to determine the range of parameters. On the other hand, elliptical model establishes a skin color distribution by using the ellipse model to determine whether the point falls in the ellipse area, thus the pixel represents the skin colour. However, because this method is based on a large number of training samples in order to determine the overall all boundary of the skin color distribution, obtaining the training data is the core of the method. More recently, Bayesian model [1] has attached much research attentions, which requires the establishment of a skin color classifier, also a large number of training sets is needed to ensure its detection rate. The Gaussian model, including a single Gaussian model [11] and a mixed Gaussian model [8], relies on the probability density formula to determine the probability of a pixel belonging to the skin point or not. Therefore, Gaussian model can accurately detect the skin area beyond the boundary of the skin area, but the speed is slow and is affected by the color space.

From the above description, we can see that a large number of body contour extraction algorithms impose strict requirement on the visual source to be processed, such as the requirement on the scene or the background of the source materials, greatly limiting the adaptive and widespread of the use of these methods. In order to meet the real-time performance of the system, this paper combines the threshold method and the ellipse model to extract the contours of the human body to achieve efficient real-time performance. The algorithm consists of three steps: a) Hair and shorts separation using a feature $\mathrm{H}$ component independent of the light changes to differentiate the hair and coat; b) Skin division by using a $\mathrm{CbCr}$ color space [2] [14] oval skin detection model to separate the 


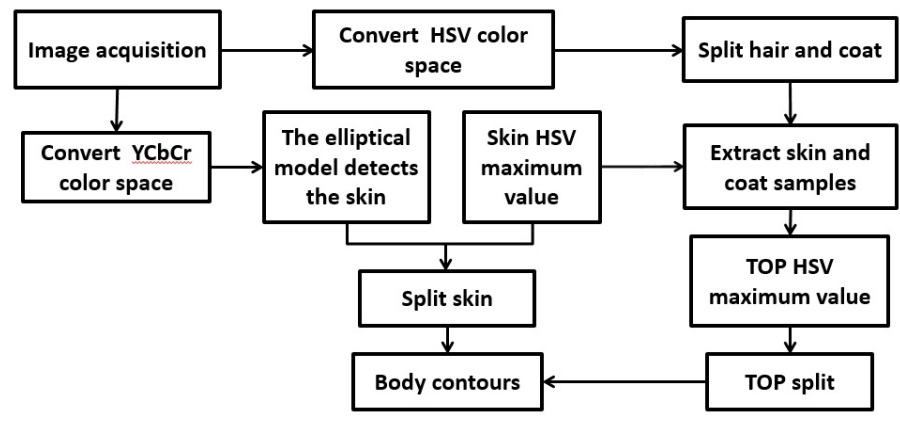

Fig. 1: Body contour extraction flow chart

skin, while automatically extracting human skin samples; extracting the sample HSV value as a threshold for better skin segmentation effect; c) T-shirt division using the threshold segmentation method to extract contour, because the shirt color is single. The algorithm is robust to the background walls and floors, and gradually extracts the contours of human hair, skin and cloth to obtain the body contour. It further reduces the backdrop and the floor, the clothe of the pre-shooting requirements. Thus, the adaptability of the algorithm has also been greatly improved.

\section{Improved Body Contour Extraction Method}

This paper introduces a robust approach for extracting contours of human body accurately from the complex background environment. Removing a background scene to sperate the human body from the scene is a difficult task due to the rapid change of the background environment. Fig 1 shows the proposed body contour extraction flow chart. The focus of this paper is on the characteristics of the human body and how to extract human body efficiently and effectively. Figure 1 outlines the extraction process, from which we can see that the main computation process for the division of clothing, hair, skin, individual differences in the body colour constitutes a very large part of this process. The use of the oval skin detection model combined with the human skin samples of HSV to maximize the effect of statistical analysis can significantly improve the skin color area recognition rate, hence, the effectiveness of the approach.

RGB images taken by a smart phone were used in the experiments. Due to photo pixels, in order to improve the processing speed and reduce the amount of redundant information, the original images were compressed to $450 \mathrm{X} 800$ resolution. As shown in Figure 2, in the experiment, a general requirement for clothing can be that participant wears a single colour vest and a dark colour shorts, and hair bundles and no shoes. The front posture of the participant was standing with feet and arms were 45 degrees open (Figure 2 (a)). The side pose was hands were vertical hanging straight (Figure 2 (b)). Bright lighting conditions was preferable, but the general home environment would be adequate. 


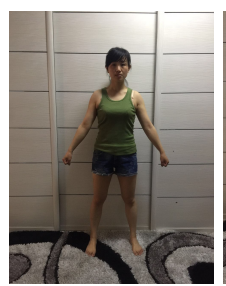

(a)

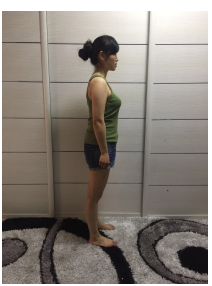

(b)

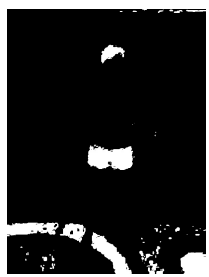

(c)

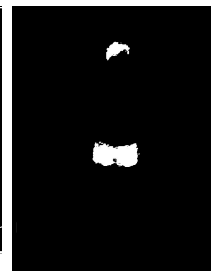

(d)

Fig. 2: RGB images (a) front image, (b)side image.(c) H binarization . (d) Processed image.

\subsection{HSV Colour Space and Segmenting Hair and Cloth}

The HSV colour space is an oriented colour coordinate system, this colour model is close to the human colour-aware simulation model [5], where $\mathrm{H}$ represents the chromaticity (a measure of the composition of the color spectrum), S represents the saturation (the pure wavelength ratio in the main wavelength), indicating the degree of the same brightness of a color distance, and V represents the purity (relative to the brightness of white light). The equation for RGB transform to HSV component is Equations 1.

$$
\begin{aligned}
& V=\max (R, G, B) \\
& S= \begin{cases}\frac{V-\min (R, G, B)}{V} & V \neq 0 \\
0 \quad & V=0\end{cases} \\
& H=\left\{\begin{array}{cc}
\frac{60 G-B}{V-\min R, G, B} & V=R \\
120+\frac{60(B-R)}{V-\min (R, G, B))} & V=G \\
240+\frac{60(B-R))}{V-\min (R, G, B))} & V=B
\end{array}\right. \\
& H=H+360, H<0
\end{aligned}
$$

In order to differentiate various parts of the human body, we must firstly analyze the HSV channels of the image and its histogram, as shown in Figure 3 . The color histogram is the colour features of the image, which describes proportions of different colours in the entire image. The advantages of using HSV channels are that these are that these are suitable for describing images that are difficult to automatically segment and do not need to consider objects spatial positions in the image.

In Figure 3, histogram peak boundaries were not clear. Therefore, directly finding the threshold of the human body from the histograms can be very difficult. Because $\mathrm{H}$ is almost independent to the changes in light, it can be used as colour comparison, so that images can be separated from the colorful color. Figure 3(a) shows hair and cloth colour contrast in $\mathrm{H}$ channel image. The threethirds of the region below the pixel value becomes 0 and the removal of the small area can be extracted. Figure 2 (d) shows the hair and shorts binary profiles. 


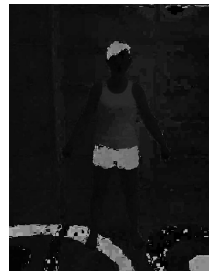

(a) $\mathrm{H}$

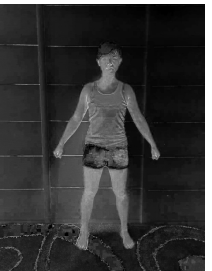

(b) $\mathrm{S}$

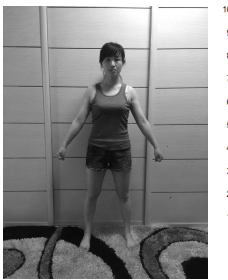

(c) $\mathrm{V}$
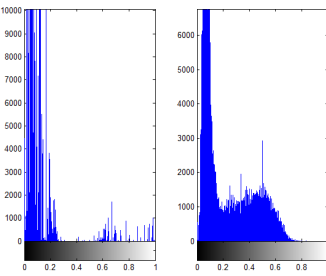

(d) histogram

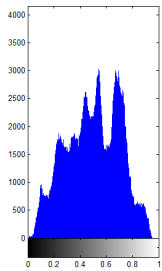

Fig. 3: HSV channel image and histogram.

\subsection{YCbCr Colour Space and Oval Skin Detection Model}

The luminance and chrominance components of the $\mathrm{YCbCr}$ colour space are separated, hence they are often used as colour models for detecting skin colours. In this mode $\mathrm{Y}$ is the brightness, $\mathrm{Cb}$ is the blue component of the light source, and $\mathrm{Cr}$ is the red component in the light source. The original RGB image conversion for $\mathrm{YCbCr}$ formula is as follows:

$$
\begin{aligned}
Y & =0.299 * R+0.587 * G+0.114 * B \\
C r & =(R-Y) * 0.713+d \\
C b & =(B-Y) * 0.564+d \\
R & =Y+1.403 *(C r-d) \\
G & =Y-0.714 *(C r-d)-0.344 *(C b-d) \\
B & =Y+1.773 *(C b-d) \\
\text { with }, d & =128,8 \text { bit } ;=32768,16 b i t ;=0.5, \text { float }
\end{aligned}
$$

The main factor in the appearance of the difference in colours is the skin colour variations of people represented as a skin colour distribution relative to the concentration in a smaller area, i.e. similar to the oval shape. The colour of the person can be converted to a $\mathrm{YCbCr}$ colour space. In the $\mathrm{CbCr}$ plane projection, we can get the skin colour sample set and KL transformation to establish the ellipse model. By determining whether the pixel $\mathrm{CbCr}$ value is in the ellipse model area, the pixel can be identified as a skin colour pixel or non-skin color pixel, separating the skin area out. The ellipse model is defined by:

$$
\frac{\left(x-e c_{x}\right)^{2}}{a^{2}}+\frac{\left(y-e c_{y}\right)^{2}}{b^{2}}=1
$$

Parameter matrix is given by:

$$
\left[\begin{array}{l}
x \\
y
\end{array}\right]=\left[\begin{array}{cc}
\cos \theta & \sin \theta \\
-\sin \theta & \cos \theta
\end{array}\right]\left[\begin{array}{l}
C^{\prime} b C_{x} \\
C^{6} r C_{y}
\end{array}\right]
$$




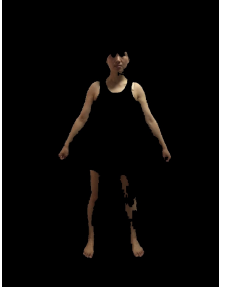

(a)

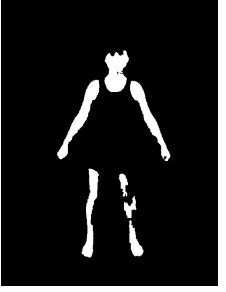

(b)

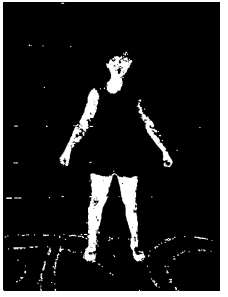

(c)

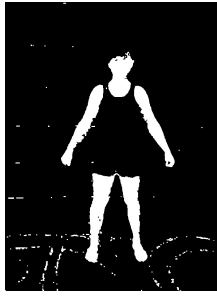

(d)

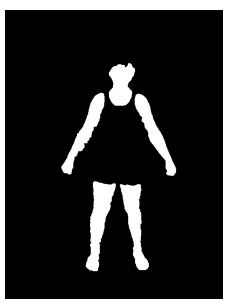

(e)

Fig. 4: Oval skin model: (a) The elliptical model for skin; (b) Ellipse models binary map.(c) Sample skin test chart; (d) Fig. 4 (b) and Fig. 4 (c) linear fusion graph;(e) Skin segmentation binary map

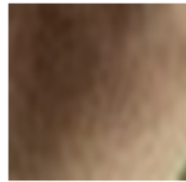

(a)

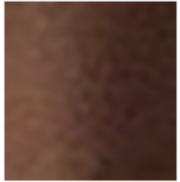

(b)

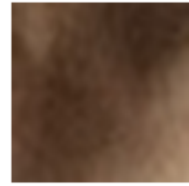

(c)

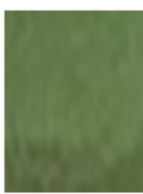

(d)

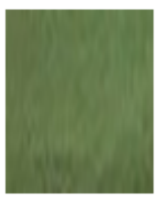

(e)

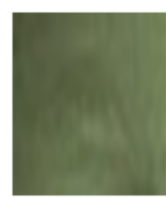

(f)

Fig. 5: Automatically extract skin((a)-(c)) and cloth samples((d)-(e))

As shown in Figure 4(a), the skin area is found to be defective, and the elliptical model does not fully recognize all the skin pixels, which is due to differences in the skin colour variations from person to person.

\subsection{Thresholds Division of The Skin Colour}

In order to solve the problem that the ellipse detection model cannot completely separate the skin, we extract the skin samples using the HSV value of the sample to determine the threshold parameters to divide the skin points. This approach helps identify the problem skin pixels and is able to mitigate the oval skin detection model deficiencies. Further, in order to define the threshold range $\theta_{i}$ for skin division, it is necessary to extract multiple sets of skin samples $S_{i}$ of the person. Define the sample Region of Interest (ROI) which specifies the upper left corner coordinates $P_{i}\left(m_{i}, n_{i}\right)$ of the rectangle and the length and the width $L_{i}$, $W_{i}$ of the rectangle. After a large number of experiments, it is concluded that the oval skin detection model of the leg and neck segmentations are not ideal for the distribution of human skin colour, we extracted three groups of samples from the left leg, right leg, and neck position to compensate the issues caused by the lighting.

After analyzing the sample histogram, the HSV maximum value of each sample is obtained, and it is set to separate the threshold. The pixels in the most significant range are marked as shown in Fig. 4 (c), then linearly merged with Fig. 4 (b) as shown in Fig. 4 (d), the extraction process must have some interfer- 


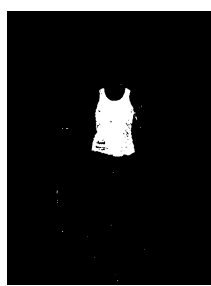

(a)

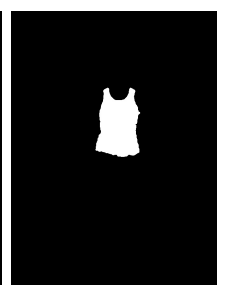

(b)

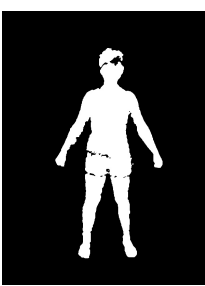

(c)

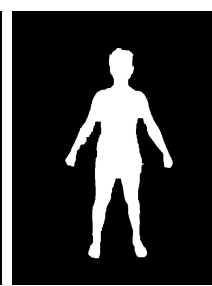

(d)

Fig. 6: (a)Linear fusion.(b) Processed image, the coat is divided. Extract the contours of the human body: (c) Integrated map; (d) Morphological operation.

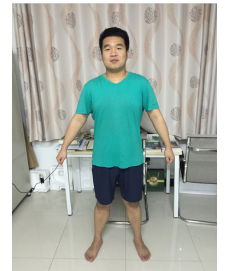

(a)

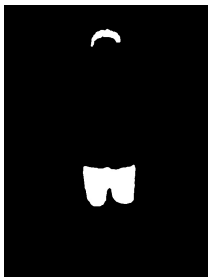

(b)

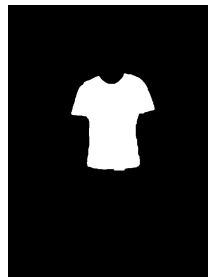

(c)

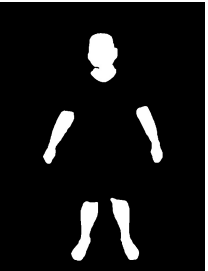

(d)

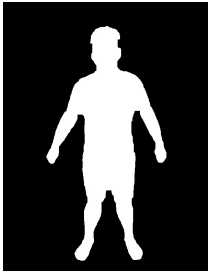

(e)

Fig. 7: another example: (a)RGB image.(b)Hair and shorts.(c) T-shirt; (d) skin.(e)Results graph.

ence points, so that removing the points and some small areas, the integration step fills the region to get more accurate results Figure 4 (e) . The method of extracting the cloth area is similar to that of skin as described above. Since the cloth colour is singular, we also extracted three areas in the middle of the image as the top cloth as shown in Fig. 5 (b). Through the analysis of the top cloth sample, the maximum value was obtained and the pixels in the most valued areas were marked out, and then the three markers were linearly merged as shown in Fig. 6 (a) to obtain the final cloth division as shown in Fig.6(b).

The final results was shown in Fig.6 (c), which was obtained by the linear fusion of the results of Fig. 2 (d), Fig. 4 (e) and Fig. 6 (b). Figure 6 (d) was obtained by morphological closing operations applied to Fig. 6(c). Figure 7 is another example of this article, the results of the segmentation is good, but also proved the feasibility of the proposed method.

\section{Conclusions and Future Work}

This paper presents an approach of body contour extraction based on the characteristics of the surface of the human body. A set of algorithms have been applied to the main areas of the cloth, hair and skin, taking into account of the large individual differences in the body colour. Based on the algorithm of elliptical skin detection model, our approach combines the two models to automatically ex- 
tract skin samples to improve the skin area Recognition rate. Finally, the results produced by the three methods were linearly merged to produce the contours via a morphological processing. Experiments show that the approach can extract the human body contours from relatively complex background scene with good results. While this work has served to propose and prototype the body extraction concept, future research will investigate algorithms to further reduce the restrictions on cloth and light and further optimization is also needed.

\section{Acknowledgement}

This work is supported by Shanxi Province Science and Technology Department of International Cooperation Projects (2016JZ026 2016KW-043) and (2016GY047)

\section{References}

1. Bouzerdoum, D.C..A.: A bayesian approach to skin color classification in ycbcr color space. In: TENCON 2000. Proceedings, 24-27 Sept. (2000)

2. Dawod A Y, Abdullah J, A.M.J.: Adaptive skin color model for hand segmentation. In: International Conference on Computer Applications and Industrial Electronics. IEEE, 486-489. (2010)

3. Deng, W., L.G.W.J.e.a.: Image extraction method of $3 \mathrm{~d}$ human body feature based on image. journal of zhejiang university, 2010, 44 (5): 837-840. Journal of Zhejiang University, 44 (5): 837-840. (2010)

4. Jiang L, Yao J, L.B.e.a.: Automatic body feature extraction from front and side images. Journal of Software Engineering \& Applications, 05(12):94-100. (2012)

5. Kaehler A, B.G.: Learning OpenCV 3 (2016)

6. Lin Y. L., Wang M., e.a.: Constructing $3 \mathrm{~d}$ human model from front and side images. Expert Systems with Applications, 39(5):5012-5018. (2012)

7. Lu, Jun-Ming, W.e.a.: Automated anthropometric data collection using $3 \mathrm{~d}$ whole body scanners. Expert Systems with Applications, 35(1-2):407-414. (2008)

8. Mokhtar M.Hasan, P.K.M.: Superior skin color model using multiple of gaussian mixture model. British Journal of Science, Vol. 6 (1) (2012)

9. Peter Peer, Jure Kovac, F.S.: Human skin color clustering for face detection. In: EUROCON 2003 (2003)

10. R, S.: Computer Vision: Algorithms and Applications. 21(8):2601-2605. (2010)

11. Subban R, M.R.: Human skin segmentation in color images using gaussian color mode. Recent Advances in Intelligent Informatics. Springer International Publishing, 13-21. (2014)

12. Teerayoot Sawangsri, Vorapoj Patanavijit, S.J.: Face segmentation using novel skin-color map and morphological technique. In: World Academy of Science, Engineering and Technology 2 (2005)

13. Yang M., Liu G., D.H.: A study on human body contour extraction method based on hsv color space. 2015 (2): 41-46. JOURNAL OF BEIJING FASHION INSTITUTE (NATURAL SCIENCE EDITION), (2015)

14. Zhang, Q.: Hand gesture segmentation based on mixed skin-color model and fcm algorithm. Journal of Information \& Computational Science,12(9):3527-3536. (2015) 